\title{
Conformation-Dependent Photophysics of Bifunctional Hydrogen Bond Donor/Acceptor Molecules
}

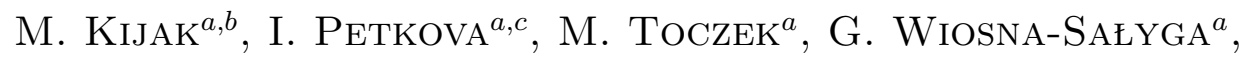 \\ A. Zielińska ${ }^{a}$, J. Herbich ${ }^{a, b}$, R.P. Thummel ${ }^{d}$ AND J. WALUK ${ }^{a, b}$ \\ ${ }^{a}$ Institute of Physical Chemistry, Polish Academy of Sciences \\ Kasprzaka 44, 01-224 Warsaw, Poland \\ ${ }^{b}$ Faculty of Mathematics and Natural Sciences, College of Science \\ Cardinal Stefan Wyszyński University, Dewajtis 5, 01-815 Warsaw, Poland \\ ${ }^{c}$ Institute of Organic Chemistry, Bulgarian Academy of Sciences \\ Akad. G. Bontchev bl. IX, Sofia 1113, Bulgaria \\ ${ }^{d}$ Department of Chemistry, University of Houston, Houston TX 77204-5003, USA
}

Dedicated to the memory of Jerzy Prochorow

A series of heteroazaaromatic molecules possessing both a hydrogen bond donor and acceptor groups linked by a single bond was studied using electronic absorption and fluorescence techniques, combined with quantum-chemical calculations. Fluorescence lifetimes and quantum yields strongly depend on the solvent. Double exponential decays are observed in alcohols. This was interpreted in terms of alcohol-induced syn-anti rotamerization in the ground electronic state. The driving force for the conformational change is provided by two factors: (a) solvent polarity increase; (b) the formation of two separate hydrogen bonds in the anti species. In the syn structure, formation of cyclic hydrogen bonds leads to rapid depopulation of the lowest excited singlet state via two competing mechanisms: proton transfer and enhanced internal conversion. The experimental results are rationalized by calculations which include specific and nonspecific solvent effects.

PACS numbers: 33.15.--e, 02.70.-c

\section{Introduction}

It is well known that the photophysical properties of a molecule may change significantly upon formation of a hydrogen bond (HB). Various mechanisms that 
may be responsible for modifications of excited state properties include electron transfer [1,2], change in the character of the emitting state [3], proximity of $n \pi^{*}$ and $\pi \pi^{*}$ levels leading to enhancement or suppression of intersystem crossing [4], phototautomerization [5] or proton displacement along the hydrogen bond [6, 7], rapid deactivation via internal conversion [8]. Our studies of numerous bifunctional azaaromatic systems, which simultaneously possess an HB donor (pyrrole NH group) and an acceptor (pyridine-type nitrogen) provided illustrations of such phenomena [9-23]. Additionally, another mechanism of altering the photophysics was found, originating in the ground electronic state [24]. In compounds composed of a donor and an acceptor linked by a single bond, syn-anti rotamerization in $S_{0}$ is possible (Fig. 1). For an appropriate topology of the donor and acceptor, the two conformers may reveal drastically different excited state properties. An example is provided by 2-(2'-pyridyl)indole $\mathbf{1}$ and related compounds, in which the excited syn form is able to form cyclic, hydrogen-bonded complexes with alcohols or water. These complexes are efficiently deactivated in $S_{1}$, via internal conversion and/or proton transfer. On the other hand, these rapid deactivation channels are not available in the anti rotamer, which cannot form cyclic complexes. As a result, the fluorescence of the anti form in protic environments is much stronger than that of the syn species.

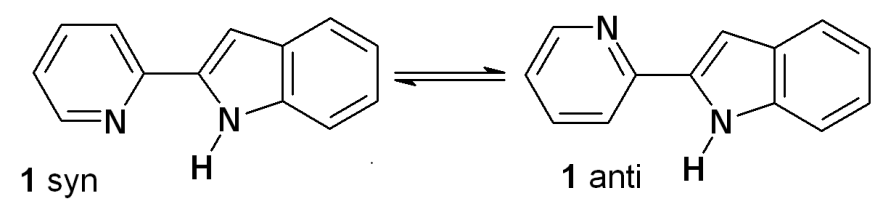

Fig. 1. Syn and anti forms of 2-(2'-pyridyl)indole (1).

The syn form is dominant in aprotic nonpolar environments. Increasing the polarity and protic abilities of the solvent leads to a relative stabilization of the anti species. This is due to two features of the anti structure: first, a larger ground state dipole moment and, second, stronger, more linear hydrogen bonds with hydroxylic partners than in the syn species. It seems that a combination of both nonspecific (stabilization in polar solvents) and specific (HB formation) effects is necessary to effectively populate the anti rotamers.

The main goal of the present work is to ascertain whether the above scheme, previously described in detail for $\mathbf{1}$ [24], can be generalized to the whole class of structurally similar molecules. The indications that it may be so have been recently obtained in the studies of 2 -(2'-pyridyl)pyrrole 2 [19], 7-( $3^{\prime}$-pyridyl)indole 3 [20], and 2-(2'-pyridyl)benzoindoles 4 and 5 [25]. We now extend the series by new compounds: 5 -methyl-2-( $2^{\prime}$-pyridyl $)$ indole $\mathbf{6}, 5$-methoxy-2-( $2^{\prime}$-pyridyl)indole $\quad 7, \quad 5$-chloro-2-( $2^{\prime}$-pyridyl)indole $\quad 8, \quad 5$-fluoro- $2-\left(2^{\prime}-\right.$ -pyridyl)indole $\quad \mathbf{9}, \quad 2$-(2'-pyrrolyl)quinoline $\quad \mathbf{1 0}, \quad$ and $\quad 2$-( $2^{\prime}$-pyrrolyl $)-1-8-$ 


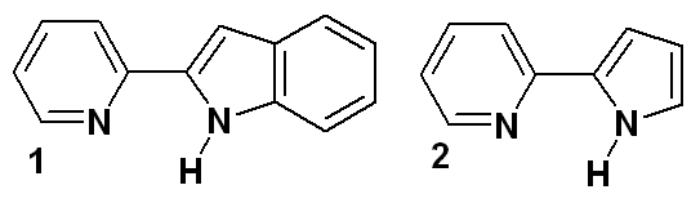<smiles>c1cncc(-c2cccc3cc[nH]c23)c1</smiles><smiles>Cc1ccnc(-c2cc3c(ccc4ccccc43)[nH]2)c1</smiles><smiles>c1ccc(-c2cc3ccc4ccccc4c3[nH]2)nc1</smiles><smiles>Cc1ccc2[nH]c(-c3ccccn3)cc2c1</smiles>

6

$\mathrm{H}^{\prime}$<smiles></smiles><smiles>Clc1ccc2[nH]c(-c3ccccn3)cc2c1</smiles><smiles>Fc1ccc2[nH]c(-c3ccccn3)cc2c1</smiles><smiles>c1c[nH]c(-c2ccc3ccccc3n2)c1</smiles><smiles></smiles>

Fig. 2. The compounds investigated in this work.

naphthyridine 11 (Fig. 2). The spectral and photophysical studies performed in protic and aprotic solvents of varying polarity confirm the validity of a model of solvent-induced syn-anti rotamerization in the ground electronic state, of which the consequences are observed in the solvent-dependent behavior of the lowest excited singlet state. A related task is to determine whether the relative energies of the syn and anti species in different environments can be reproduced by calculations. For this purpose, energies of selected molecules are computed, taking into account solvent properties.

\section{Experimental and theoretical procedures}

The syntheses and purification procedures have been described separately for $\mathbf{1}, \mathbf{1 0}$, and $\mathbf{1 1}$ [26], $\mathbf{2}$ [19], $\mathbf{3}$ [27], 4, $\mathbf{5}$ [25], $\mathbf{6}$ [28], $\mathbf{7}$ [29], and 8-9 [30]. Spectral grade solvents: $n$-hexane (HX), acetonitrile (ACN) (Aldrich), and propanol-1 
(PrOH) (Merck) were checked for the presence of fluorescing impurities. Electronic absorption spectra were recorded on a Shimadzu UV 3100 spectrophotometer. Stationary fluorescence spectra were measured using an Edinburgh FS 900 CDT spectrofluorimeter. Quinine sulphate in $0.05 \mathrm{M} \mathrm{H}_{2} \mathrm{SO}_{4}$ was used as a standard $\left(\varphi_{\mathrm{fl}}=0.51\right)$ for the determination of fluorescence quantum yields.

Fluorescence lifetimes were measured on an Edinburgh FL 900 CDT time-resolved fluorometer. Time-resolved single photon counting technique was used, followed by data reconvolution using non-linear least squares fitting routines.

Geometry optimizations, calculations of solvent-induced energy shifts, and electronic transition energies were performed using Gaussian 03 [31]. The models/basis sets included B3LYP/6-31+G(d,p), B3LYP/6-311+G(d,p), and HF/6$31+\mathrm{G}(\mathrm{d}, \mathrm{p})$. Vertical excitation energies were obtained using time-dependent density functional theory (TDDFT), the B3LYP functional, and 6-31+G(d,p) or $6-311+\mathrm{G}(\mathrm{d}, \mathrm{p})$ basis sets. The calculations, which included the presence of a solvent, used either the Onsager dipole model [32-36] or a polarizable continuum model (PCM) [37-39]. In the calculations of relative stabilities of alcohol complexes, the basis set superposition error was taken into account by using the counterpoise correction $[40,41]$.

\section{Results and discussion}

Figures $3-5$ show examples of absorption and fluorescence spectra obtained for $\mathbf{2}, \mathbf{8}$, and $\mathbf{1 1}$ in different solvents: nonpolar ( $n$-hexane), polar aprotic (acetonitrile), and protic (propanol-1). The absorption/fluorescence patterns are similar in each compound, except $\mathbf{2}$, which shows dual fluorescence in nonpolar solvents. The values of quantum yields and fluorescence decay times are presented in Table I. Table II contains the energies of the maxima of the absorption and fluorescence bands; it also includes the vertical transition energies calculated for the syn forms.

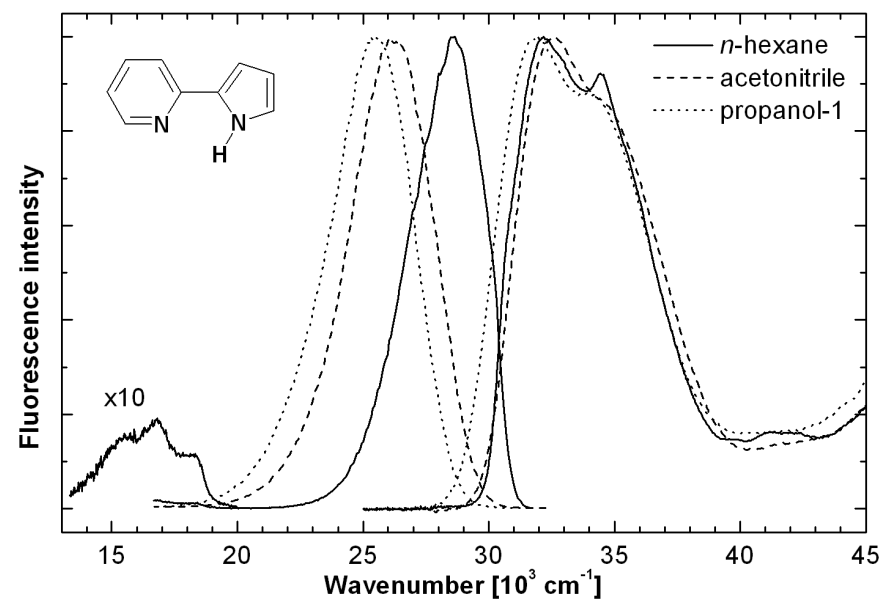

Fig. 3. Absorption and fluorescence of $\mathbf{2}$ in $n$-hexane, acetonitrile, and propanol- 1 at $293 \mathrm{~K}$. 


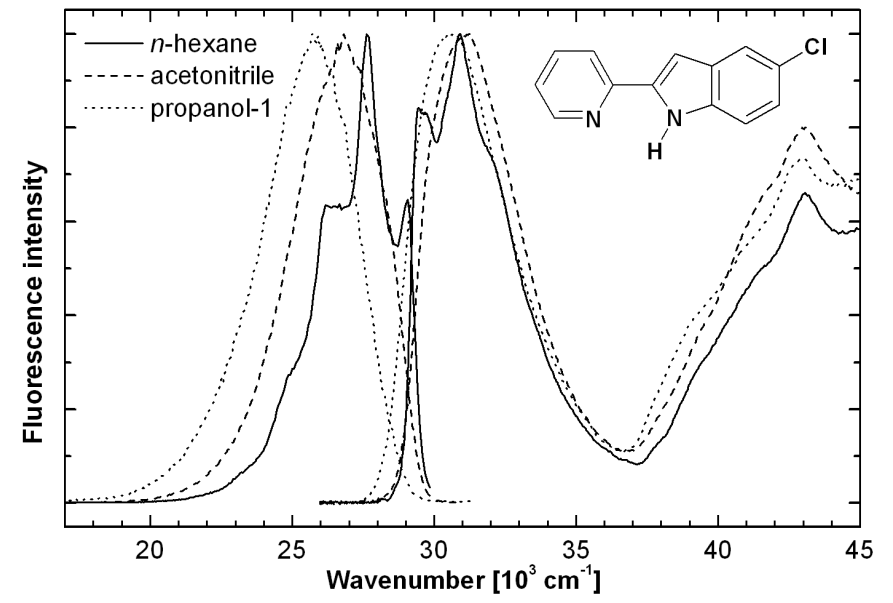

Fig. 4. Absorption and fluorescence of $\mathbf{8}$ in $n$-hexane, acetonitrile, and propanol- 1 at $293 \mathrm{~K}$.

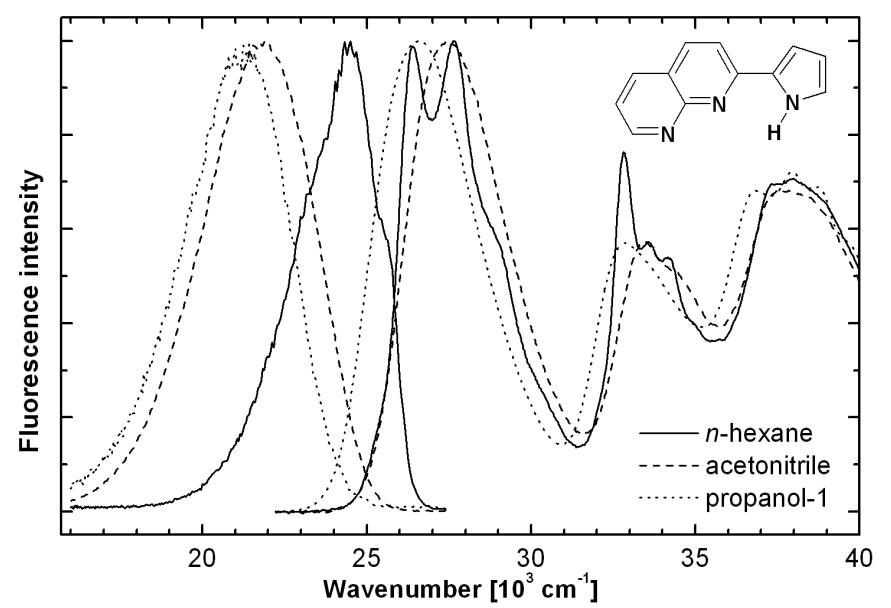

Fig. 5. Absorption and fluorescence of $\mathbf{1 1}$ in $n$-hexane, acetonitrile, and propanol-1 at $293 \mathrm{~K}$.

The photophysical data reveal a general tendency: the fluorescence quantum yields are quite high in aprotic solvents, but become significantly lower in alcohol solutions. The three exceptions to this rule are provided by $n$-hexane solutions of $\mathbf{2}, \mathbf{1 0}$, and 11. As reported elsewhere [19, 21], low fluorescence intensity in $\mathbf{2}$ is due to the excited state intramolecular proton transfer, leading to a short-lived phototautomer. The presence of the latter is manifested by the appearance of a low-energy fluorescence band emitted from the excited tautomeric form (Fig. 3). In turn, the low fluorescence quantum yields in $\mathbf{1 0}$ and $\mathbf{1 1}$, the compounds containing quinoline and diazanaphthalene moieties, may be due to the presence of 
TABLE I

Fluorescence quantum yields and lifetimes obtained in various solvents at $293 \mathrm{~K}$.

\begin{tabular}{c|c|c|c|c|c|c}
\hline \hline \multirow{2}{*}{} & \multicolumn{2}{|c|}{$n$-Hexane } & \multicolumn{2}{c|}{ Acetonitrile } & \multicolumn{2}{c}{ Propanol- } \\
\cline { 2 - 7 } & $\varphi_{\mathrm{fl}}{ }^{a}$ & $\tau_{\mathrm{fl}}{ }^{b}$ & $\varphi_{\mathrm{fl}}$ & $\tau_{\mathrm{fl}}$ & $\varphi_{\mathrm{fl}}$ & $\tau_{\mathrm{fl}}$ \\
\hline $\mathbf{1}$ & 0.48 & 1.3 & 0.53 & 1.8 & 0.11 & $<0.2 / 3.2$ \\
$\mathbf{2}$ & 0.008 & $0.033^{c}$ & 0.15 & $0.6 / 4.3$ & 0.12 & 4.1 \\
$\mathbf{3}$ & 0.21 & 1.1 & 0.25 & $3.4 / 8.7$ & 0.0025 & $0.2 / 2.9$ \\
$\mathbf{4}$ & 0.72 & 1.3 & 0.79 & 1.6 & 0.25 & $<0.1 / 2.6$ \\
$\mathbf{5}$ & 0.40 & 1.4 & 0.51 & 1.7 & 0.24 & $<0.1 / 2.6$ \\
$\mathbf{6}$ & 0.44 & 1.1 & 0.52 & 2.2 & 0.15 & $<0.1 / 3.3$ \\
$\mathbf{7}$ & 0.17 & 0.9 & 0.29 & 2.6 & 0.04 & $<0.1 / 1.6$ \\
$\mathbf{8}$ & 0.35 & 0.9 & 0.40 & 1.1 & 0.095 & $<0.1 / 1.4$ \\
$\mathbf{9}$ & 0.43 & 1.2 & 0.56 & 1.8 & 0.12 & 2.4 \\
$\mathbf{1 0}$ & 0.059 & $\approx 0.2$ & 0.33 & 2.4 & 0.058 & $<0.2 / 3.4$ \\
$\mathbf{1 1}$ & 0.048 & $\approx 0.2$ & 0.55 & 4.6 & 0.043 & $<0.2 / 5.0$ \\
\hline
\end{tabular}

${ }^{a}$ estimated accuracy: $\pm 20 \%$; ${ }^{b}$ lifetimes in ns; slashes separate two values in the case of biexponential decays; ${ }^{c}$ measured with picosecond resolution [46]

low-lying $n \pi^{*}$ states, both in the singlet and triplet manifolds. The interactions between close-lying $n \pi^{*}$ and $\pi \pi^{*}$ levels can enhance both the intersystem crossing to the triplet state and $S_{0} \leftarrow S_{1}$ internal conversion (the "proximity effects" $[42,43])$. Upon passing to polar acetonitrile, these effects disappear, as the $n \pi^{*}$ states are destabilized relative to the $\pi \pi^{*}$ levels.

The most interesting observation is the biexponential fluorescence decay observed in alcohol solutions (and, for $\mathbf{2}$ and $\mathbf{3}$, in acetonitrile). Using a combination of NMR, UV-VIS electronic spectroscopy, and calculations, it has been demonstrated for 1 that the two lifetimes correspond to the presence in alcohols of both syn and anti tautomeric forms [24]. Only the syn species can form cyclic complexes with alcohols. Such complexes can be rapidly deactivated via enhanced $S_{0} \leftarrow S_{1}$ internal conversion. The longer-lived component is therefore assigned to the anti form. The present results show that the phenomenon is quite general and insensitive to structural details. For instance, the same effect of alcohol is observed for $\mathbf{1}$ and 10, constitutional isomers which can be obtained from $\mathbf{2}$ by fusing a phenyl ring to either the pyrrole or pyridine rings.

Again, the behavior of $\mathbf{2}$ differs from the other compounds in that the biexponential decay is observed in acetonitrile, not in alcohols. This makes sense when one notes that $\mathbf{2}$ is the smallest of all the compounds studied, and, therefore should possess the smallest Onsager radius. Therefore, it should reveal the largest relative stabilization of the more polar rotameric form in acetonitrile with respect 
TABLE II

TDDFT-calculated vertical $S_{0} \rightarrow S_{1}$ transition energies and the values of absorption and fluorescence maxima obtained in various solvents at $293 \mathrm{~K}$.

\begin{tabular}{c|c|c|c|c|c|c|c}
\hline \hline & Calculated & \multicolumn{2}{|c|}{$n$-Hexane } & \multicolumn{2}{|c|}{ Acetonitrile } & \multicolumn{2}{|c}{ Propanol-1 } \\
\cline { 3 - 7 } & transition en. $^{a}$ & $\tilde{\nu}_{\text {abs }}^{b}$ & $\tilde{\nu}_{\mathrm{f}}^{c}$ & $\tilde{\nu}_{\text {abs }}$ & $\tilde{\nu}_{\mathrm{fl}}$ & $\tilde{\nu}_{\text {abs }}$ & $\tilde{\nu}_{\mathrm{fl}}$ \\
\hline $\mathbf{1}$ & 29.9 & 30.8 & 27.5 & 31.0 & 26.5 & 30.4 & 24.6 \\
$\mathbf{2}$ & 32.3 & 32.2 & 28.7 & 32.6 & 26.3 & 31.9 & 25.5 \\
& & & $16.8^{e}$ & & & & \\
$\mathbf{3}$ & 32.5 & 33.6 & 28.9 & 33.5 & 25.9 & 32.9 & 26.2 \\
$\mathbf{4}$ & $28.7^{d}$ & 27.6 & 26.1 & 27.9 & 26.0 & 27.6 & 24.8 \\
$\mathbf{5}$ & $29.0^{d}$ & 27.6 & 26.1 & 27.9 & 26.0 & 27.6 & 25.3 \\
$\mathbf{6}$ & 29.7 & 30.6 & 27.3 & 31.0 & 26.1 & 30.3 & 24.8 \\
$\mathbf{7}$ & $28.3 / 30.1^{f}$ & 30.8 & 26.3 & 30.9 & 24.6 & 30.0 & 24.5 \\
$\mathbf{8}$ & 30.2 & 30.9 & 27.6 & 31.1 & 26.7 & 30.6 & 25.8 \\
$\mathbf{9}$ & 30.2 & 30.9 & 27.6 & 31.3 & 26.8 & 30.7 & 25.6 \\
$\mathbf{1 0}$ & 27.7 & $27.4 / 28.7^{g}$ & 25.6 & 28.4 & 23.8 & 28.0 & 23.3 \\
$\mathbf{1 1}$ & 26.9 & $26.4 / 27.6^{g}$ & 24.5 & 27.5 & 21.8 & 26.6 & 21.2 \\
\hline
\end{tabular}

${ }^{a} \mathrm{~B} 3 \mathrm{LYP} / 6-31+\mathrm{G}(\mathrm{d}, \mathrm{p})$ ground state optimized geometry, the values in $10^{3} \mathrm{~cm}^{-1} ;{ }^{b}$ maximum of the lowest energy absorption band; ${ }^{c}$ maximum of fluorescence; ${ }^{d} 6-31 \mathrm{G}(\mathrm{d}, \mathrm{p})$ basis set; ${ }^{e}$ emission of the phototautomer; ${ }^{f}$ much larger oscillator strength calculated for the higher transition; ${ }^{g}$ two bands of comparable intensity

to $n$-hexane. The experiment shows clearly that it is the anti form which is more polar in the ground state. Besides $\mathbf{2}$, a single exponential decay is also obtained in propanol for 9. Two different explanations can be proposed. First, single exponential decay could indicate that only one (anti) form is present. However, this would imply that the radiative constant in the anti form is much smaller than that of the syn species. Such assumption is hard to reconcile with the results of calculations, which predict similar oscillator strengths for low-energy transitions in both rotamers. A more plausible interpretation is that the syn forms are still present, but their excited states are deactivated too rapidly to be detected with only subnanosecond resolution. We recall that the $S_{1}$ depopulation in cyclic alcohol complexes of the syn species may involve not only enhanced internal conversion, but also excited state proton transfer [18]. The latter process occurs in cyclic 1:1 complexes on the timescale of single picoseconds or faster, whereas the former involves complexes with two molecules of alcohol, and takes much longer (tens to hundreds of picoseconds) [16]. The equilibrium between 1:1 and 1:2 complexes strongly depends on the peripheral structure of the chromophore $[14,15]$. The present results would at first suggest that in $\mathbf{2}$ and $\mathbf{9}$ the equilibrium is dominated 
by the 1:1 species that, upon excitation undergo fast phototautomerization. However, we cannot exclude that for these particular molecules the 1:2 complexes are also rapidly deactivated. As discussed below, both experiment and theory indicate that syn 1:2 structures may be dominant.

We should note that the error in the determination of the relative contributions of the two emissions becomes very large if one of the decays is too short to be obtained exactly; this is the reason why the amplitudes were not included in Table I. When the two components of the biexponential decay are determined reliably, one can estimate the ground state fractions of the syn and anti rotamers using relative amplitudes. This has been done for $\mathbf{2}$ in acetonitrile, assuming similar extinction coefficients and absorption spectra of the two forms. The contributions of the two decay components do not vary strongly across the fluorescence band ( $85 \%$ contribution of the syn emission at $28571 \mathrm{~cm}^{-1}, 79 \%$ at $26455 \mathrm{~cm}^{-1}$, and $76 \%$ at $24390 \mathrm{~cm}^{-1}$ ). These values result in the fraction of the anti form ranging between $2.4-4.2 \%$. Interestingly, for excitation at the red edge of the first absorption band, the contribution from the anti population increases significantly, showing that the absorption of the anti species is red-shifted with respect to the syn rotamer. The analogous procedure applied to $\mathbf{3}$ in acetonitrile yielded $4 \%$ as the estimated fraction of the anti form.

Because of the presence of two different rotameric structures with very different $S_{1}$ lifetimes and quantum yields, the values of the quantum yields, $\varphi_{\text {obs }}$, obtained for cases of biexponential decay (Table I) should be treated with caution. It should be stressed that the yields measured for alcohols provide only a lower limit for $\varphi_{\text {anti }}$, the fluorescence quantum yield of the anti form $\left(\varphi_{\text {obs }}=\left(1-f_{\text {anti }}\right) \varphi_{\text {syn }}+f_{\text {anti }} \varphi_{\text {anti }}\right.$, where $f_{\text {anti }}$ is the fraction of the anti forms in the excited state). Thus, the real values of quantum yields of the H-bonded anti forms can, in principle, be even higher than the yields of the non-H-bonded syn species. Such possibility is also confirmed by the observation of longer decay time values in alcohols than in acetonitrile.

In order to check to what extent the experimental results can be quantitatively reproduced by theory, we calculated the relative energies of syn and anti forms along with the corresponding ground state dipole moments. They are presented in Table III. It can be seen that the calculations predict very similar syn-anti energy differences for isolated molecules. The anti forms have energies 4-6 kcal/mol higher than the syn rotamers in all compounds except $\mathbf{3}$, where the two forms lie very close in energy. The different behaviour of $\mathbf{3}$ can be explained by the fact that it is the only molecule where the HB donor and acceptor moieties are twisted against each other by a large angle (about $45^{\circ}$ ) in both rotameric forms. For most cases, the ground state dipole moments are significantly larger for the anti species, which suggests that this form should be preferentially stabilized in polar solvents. Therefore, the next step was to estimate the amount of this stabilization. Various approaches have been tried for selected molecules: a simple 
TABLE III

B3LYP-calculated ground state dipole moments $\left(\mu_{\mathrm{g}}\right)$, relative energies of the syn and anti forms $\left(\Delta E=E_{\text {anti }}-E_{\mathrm{syn}}\right)$ and the changes in the relative energies due to solvation $\left(\Delta E_{\text {solv }}\right)$, obtained using the PCM model.

\begin{tabular}{c|c|c|c|c|c|c|c}
\hline \hline & \multicolumn{2}{|c|}{$\mu_{\mathrm{g}}[\mathrm{D}]$} & $\Delta E^{a}$ & \multicolumn{2}{|c|}{$\Delta E_{\text {solv }}[\mathrm{kcal} / \mathrm{mol}]$} & \multirow{2}{*}{ Basis set/method $^{b}$} \\
\cline { 5 - 7 } & syn & anti & {$[\mathrm{kcal} / \mathrm{mol}]$} & $n$-Heptane & ACN & Ethanol & \\
\hline $\mathbf{1}$ & 2.44 & 3.44 & 4.10 & -0.59 & -2.30 & -2.21 & $6-31+\mathrm{G}(\mathrm{d}, \mathrm{p}) / \mathrm{Opt}$ \\
$\mathbf{2}$ & 1.06 & 3.22 & 4.41 & -0.72 & -2.57 & -2.49 & $6-31+\mathrm{G}(\mathrm{d}, \mathrm{p}) / \mathrm{Opt}$ \\
$\mathbf{3}$ & 1.63 & 2.98 & 0.26 & -0.05 & -0.25 & -0.34 & $6-311+\mathrm{G}(\mathrm{d}, \mathrm{p}) / \mathrm{SP}$ \\
$\mathbf{4}$ & 2.28 & 3.50 & 4.26 & -0.51 & -2.38 & -2.29 & $6-31 \mathrm{G}(\mathrm{d}, \mathrm{p}) / \mathrm{SP}$ \\
$\mathbf{5}$ & 2.27 & 3.05 & 4.39 & -0.56 & -2.38 & -2.29 & $6-31 \mathrm{G}(\mathrm{d}, \mathrm{p}) / \mathrm{SP}$ \\
$\mathbf{6}$ & 1.98 & 3.11 & 4.13 & -0.60 & -2.34 & -2.26 & $6-31+\mathrm{G}(\mathrm{d}, \mathrm{p}) / \mathrm{SP}$ \\
$\mathbf{7}$ & 2.73 & 2.55 & 4.11 & -0.62 & -2.30 & -2.22 & $6-31+\mathrm{G}(\mathrm{d}, \mathrm{p}) / \mathrm{SP}$ \\
$\mathbf{8}$ & 4.73 & 5.63 & 4.29 & -0.63 & -2.42 & -2.33 & $6-31+\mathrm{G}(\mathrm{d}, \mathrm{p}) / \mathrm{SP}$ \\
$\mathbf{9}$ & 4.37 & 5.34 & 4.21 & -0.54 & -2.33 & -2.24 & $6-31+\mathrm{G}(\mathrm{d}, \mathrm{p}) / \mathrm{SP}$ \\
$\mathbf{1 0}$ & 0.83 & 3.09 & 4.76 & -0.67 & -2.49 & -2.40 & $6-31+\mathrm{G}(\mathrm{d}, \mathrm{p}) / \mathrm{Opt}$ \\
$\mathbf{1 1}$ & 2.58 & 5.15 & 5.61 & -0.80 & -3.38 & -3.26 & $6-31+\mathrm{G}(\mathrm{d}, \mathrm{p}) / \mathrm{SP}$ \\
\hline \multicolumn{1}{c}{ ZPE }
\end{tabular}

${ }^{a} \mathrm{ZPE}$ corrected; ${ }^{b} \mathrm{Opt}$ - geometry optimized using PCM, SP — optimized vacuum geometry used

Onsager model [36], its implementation in Gaussian [32-35] and the PCM [37-39]. Since $n$-hexane and propanol-1 are not implemented for PCM in Gaussian, they were replaced by $n$-heptane and ethanol. The results are shown in detail for $\mathbf{1}$, 2, and $\mathbf{1 0}$ in Table IV. All procedures that use the PCM model yield very similar relative stabilization energies for the anti form. The geometry reoptimization in the solvent environment leads to only minor changes in relative energies. Even the replacement of DFT by the HF model does not significantly alter the results. Therefore, the remaining compounds, presented in Table III, were computed using a simplified PCM DFT/single-point approach, which did not involve geometry reoptimization in the $\mathrm{PCM}$ procedure.

The results presented in Table III indicate that the relative stabilization of the more polar anti form by a polar solvent is not sufficient to reverse the energy ordering of the two rotamers. The quantitative comparison with experiment can be performed for $\mathbf{2}$ in acetonitrile. The calculations place the syn form in acetonitrile about $1.8 \mathrm{kcal} / \mathrm{mol}$ below the anti rotamer. This corresponds to $4.6 \%$ of the anti form at room temperature, in excellent agreement with the experimental estimation of 2-4\%. For other compounds, the calculated anti-syn energy differences are comparable: the predicted fractions of the anti forms are about $2-5 \%$. In order to understand why a small fraction of the anti form is readily observed in acetonitrile for $\mathbf{2}$, but not for other compounds (except $\mathbf{3}$ ) we note that the fluorescence quantum yield of the syn rotamer of $\mathbf{2}$ is small, due to intramolecular 
TABLE IV

Calculated changes in the relative energies of the syn and anti rotamers using various approaches. See Table III for details.

\begin{tabular}{|c|c|c|c|c|c|c|c|c|c|}
\hline & \multicolumn{2}{|c|}{$\mu_{\mathrm{g}}[\mathrm{D}]$} & \multirow{2}{*}{$\begin{array}{c}\Delta E \\
{\left[\frac{\mathrm{kcal}}{\mathrm{mol}}\right]} \\
\end{array}$} & \multicolumn{5}{|c|}{$\Delta E_{\text {solv }}[\mathrm{kcal} / \mathrm{mol}]$} & \multirow[t]{2}{*}{ 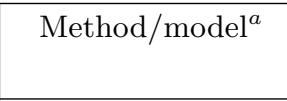 } \\
\hline & syn & anti & & $\mathrm{HPT}^{b}$ & $\mathrm{HX}$ & $\mathrm{ACN}$ & $\mathrm{EtOH}^{c}$ & $\mathrm{rOH}$ & \\
\hline 1 & 2.44 & 3.56 & 4.04 & $\begin{array}{l}-0.59 \\
-0.60 \\
-0.56 \\
-0.38 \\
\end{array}$ & -0.38 & $\begin{array}{l}-2.30 \\
-2.34 \\
-2.07 \\
-0.94 \\
\end{array}$ & $\begin{array}{l}-2.21 \\
-2.26 \\
-2.00 \\
-0.96 \\
\end{array}$ & -0.94 & $\begin{array}{c}\mathrm{PCM} / \mathrm{DFT} / \mathrm{Opt} \\
\mathrm{PCM} / \mathrm{DFT} / \mathrm{SP} \\
\mathrm{PCM} / \mathrm{HF} / \mathrm{SP} \\
\text { Onsager/DFT/Opt }\end{array}$ \\
\hline 2 & 1.54 & 3.38 & 4.41 & $\begin{array}{l}-0.72 \\
-0.73 \\
-0.69 \\
-0.70 \\
-0.67 \\
-0.67 \\
\end{array}$ & $\begin{array}{l}-0.65 \\
-0.65 \\
\end{array}$ & $\begin{array}{l}-2.57 \\
-2.65 \\
-2.51 \\
-2.53 \\
-2.16 \\
-2.17 \\
\end{array}$ & $\begin{array}{l}-2.49 \\
-2.55 \\
-2.42 \\
-2.44 \\
-2.10 \\
-2.11 \\
\end{array}$ & $\begin{array}{l}-2.06 \\
-2.07 \\
\end{array}$ & $\begin{array}{c}\mathrm{PCM} / \mathrm{DFT} / \mathrm{Opt} \\
\mathrm{PCM} / \mathrm{DFT} / \mathrm{SP} \\
\mathrm{PCM} / \mathrm{HF} / \mathrm{Opt} \\
\mathrm{PCM} / \mathrm{HF} / \mathrm{SP} \\
\text { Onsager/DFT/Opt } \\
\text { Onsager/DFT/SP }\end{array}$ \\
\hline 10 & 1.29 & 3.30 & 4.76 & $\begin{array}{l}-0.67 \\
-0.68 \\
-0.67 \\
-0.57\end{array}$ & -0.56 & $\begin{array}{r}-2.49 \\
-2.57 \\
-2.43 \\
-1.82\end{array}$ & $\begin{array}{l}-2.40 \\
-2.48 \\
-2.35 \\
-1.77\end{array}$ & -1.74 & $\begin{array}{c}\mathrm{PCM} / \mathrm{DFT} / \mathrm{Opt} \\
\mathrm{PCM} / \mathrm{DFT} / \mathrm{SP} \\
\mathrm{PCM} / \mathrm{HF} / \mathrm{SP} \\
\text { Onsager/DFT/Opt }\end{array}$ \\
\hline
\end{tabular}

${ }^{a} 6-31+\mathrm{G}(\mathrm{d}, \mathrm{p})$ basis set; B3LYP functional used in DFT calculations; Opt - optimized using PCM, SP — optimized vacuum geometry used; ${ }^{b} n$-heptane; ${ }^{c}$ ethanol

excited state proton transfer. Therefore, the contribution of the emission from the syn form relative to the fluorescence of the anti species is much smaller than for other compounds, which do not undergo intramolecular phototautomerization. As a result, a minor fraction of the anti species can be detected in $\mathbf{2}$ because their fluorescence is relatively strong. This is not the case with the other compounds, which seem to have comparable fluorescence quantum yields for both rotamers.

In hydroxylic solvents, the other factor that can change the relative energies of the syn and anti forms is the strength of hydrogen bonds in the two rotamers. We have performed the calculations for $\mathbf{1}$ and $\mathbf{2}$, comparing various types of complexes with methanol. Cyclic complexes of the syn form of 1:1 stoichiometry, cyclic 1:2, and cyclic 1:1 solvates with another alcohol molecule attached were considered. For the anti form, a 1:2 complex, involving independent, linear hydrogen bonds with the donor and acceptor groups was taken into account. In order to compare relative stabilities of various complexes, the energies of one or two methanol molecules, or of an optimized methanol dimer were subtracted from the energy of a complex. The calculations, somehow refined with respect to those reported previously for $\mathbf{2}$ [19], predict that, after correcting for zero-point energies (ZPE), the 1:2 anti and the cyclic 1:2 syn complexes (Fig. 6) are stabilized practically to the 


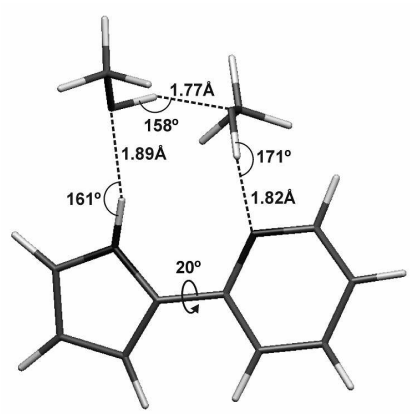

syn

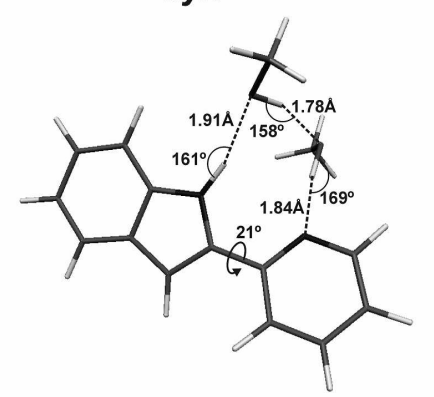

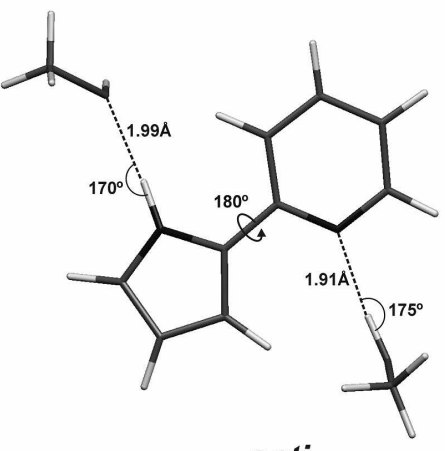

anti

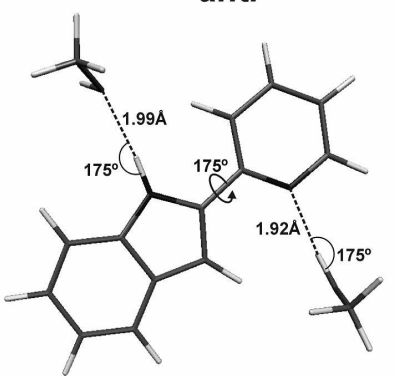

Fig. 6. Optimized structures of 1:2 complexes of the syn and anti rotamers of $\mathbf{1}$ (bottom) and $\mathbf{2}$ (top) with methanol.

same degree due to hydrogen bond formation (the stabilization is slightly larger in the anti form, by 0.4 and $0.1 \mathrm{kcal} / \mathrm{mol}$ for $\mathbf{1}$ and $\mathbf{2}$, respectively). Interestingly, without the ZPE correction the anti structure is favoured by about $1 \mathrm{kcal} / \mathrm{mol}$ (1.4 in $\mathbf{1}, 1.0$ in $\mathbf{2}$ ). The experimental results obtained for the whole group of compounds indicates that the latter prediction is closer to reality. Most probably, the errors associated with using harmonic approximation in calculations of vibrations involved in hydrogen bonds of the complexes and of the alcohol dimer lead to unbalanced ZPE corrections (it should be noted that we subtract the vibrational component of the methanol dimer only in the case of the syn complex). We conclude that the protic environment leads to a further stabilization of the anti form. The two rotamers now can have comparable energies and should therefore be detectable. This prediction is nicely corroborated by the observation of two forms in alcohols.

We have also calculated, using TDDFT, the transition energies for both rotameric forms. The results indicate that the lowest transitions should have comparable energies for both species. The calculated $S_{0}-S_{1}$ transition energies are, in general, in perfect agreement with the experimentally measured absorption maxima (Table II); the largest discrepancy does not exceed $1500 \mathrm{~cm}^{-1}$. For some cases, predictions of solvent shifts could be made, based on the calculated $S_{1}$ dipole 
moments. For instance, the TDDFT calculations for the syn forms of $\mathbf{2}, \mathbf{4}$, and $\mathbf{5}$ predict that, upon excitation to $S_{1}$, the dipole moment increases, and its direction is reversed. Such situation should lead to a blue shift in polar solvents. This is indeed observed.

TDDFT/B3LYP/6-31+G(d,p) calculations of transition energies in $\mathbf{1 0}$ and 11 confirmed the hypothesis of low-lying $n \pi^{*}$ states. In $\mathbf{1 0}$ an $n \pi^{*}$ triplet state was computed to be nearly isoenergetic with $S_{1}\left(\pi \pi^{*}\right)\left(28150\right.$ and $28170 \mathrm{~cm}^{-1}$, respectively). For 11, an $n \pi^{*}$ triplet level was predicted to lie about $1000 \mathrm{~cm}^{-1}$ above $S_{1}$. These results suggest that relatively low values of fluorescence quantum yield of $\mathbf{1 0}$ and $\mathbf{1 1}$ in $n$-hexane are due to a rapid $S_{1} \rightarrow T_{1}$ intersystem crossing.

\section{Summary}

The experimental and theoretical results obtained for a series of compounds composed of $\mathrm{HB}$ donor and acceptor moieties linked by a single bond allow us to formulate a general model for solvent-induced rotamerization (Fig. 7). Both specific (hydrogen bonding) and nonspecific (stabilization in a polar solvent) effects contribute to the change in ground state equilibrium between the syn and anti forms. In nonpolar solvents only the syn species should be present (with, perhaps, 3 being an exception). A small fraction of the anti form can be expected in polar aprotic solvents; this fraction should further increase in alco-

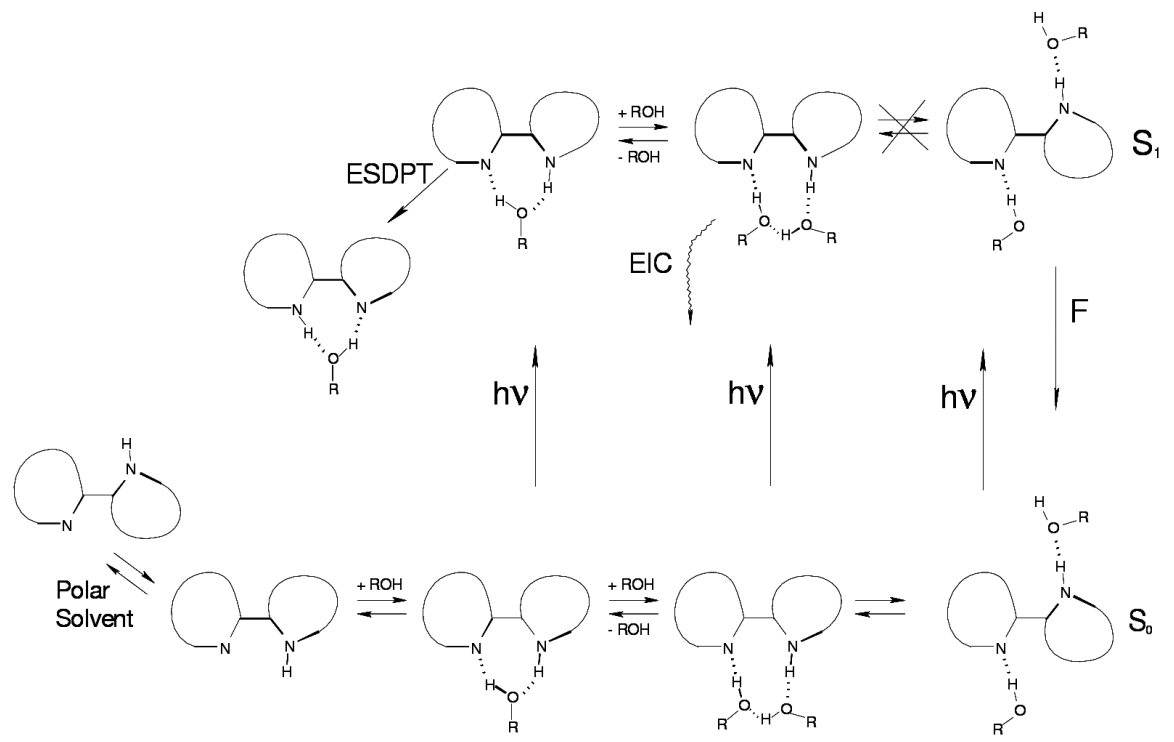

Fig. 7. Different rotamers, complexes, and tautometric forms. Bottom - ground state equilibria, top - the main channels of $S_{1}$ depopulation in alcohol complexes: ESDPT - excited state double proton transfer; EIC - enhanced internal conversion, F fluorescence. 
hols. The syn-anti conversion may have a dramatic influence on the photophysical properties if different excited state depopulation channels are operative in the two forms, as is the case of the presently studied molecules. The alcohol complexes of the syn forms undergo a rapid excited state double proton transfer (ESDPT) in 1:1 cyclic complexes and enhanced internal conversion (EIC) in 1:2 species. The latter process can actually be considered as "aborted" phototautomerization involving 3 protons [23]. In rigid bifunctional azaaromatics, such as $1 H$-pyrrolo[3,2- $h]$ quinoline, dipyrido[2,3- $\left.a: 3^{\prime}, 2^{\prime}-i\right]$ carbazole or $3,3^{\prime}$-dimethylene-2-( $2^{\prime}$-pyridyl)indole, the two types of complexes can be readily distinguished, both kinetically and spectrally (appearance of tautomeric fluorescence with the rise time characteristic of the decay of $1: 1$ species) $[11,14,16]$. The lack of tautomeric fluorescence in alcohol solutions for all the compounds studied in this work is a strong indication that 1:2 complexes dominate. Most probably, the conformational flexibility, due to the single bond between the HB donor and acceptor moieties allows a low energy structure to be achieved in the complex of the syn rotamer with two alcohol molecules. The calculations performed for $\mathbf{1}$ and 2 confirm such a hypothesis.

Possible applications of solvent-induced effects can be envisaged, for instance using the rotamerizing chromophores as indicators of local polarity/hydrophilicity (e.g., in "wet" and "dry" protein pockets).

The results of calculations show that the theory is now able to quite reliably predict rotameric equilibria, and, what seems even more important, solvent-induced changes in these equilibria, due to nonspecific effects. While the almost perfect agreement obtained for 2-(2'-pyridyl)pyrrole in acetonitrile may be fortuitous, we estimate that the accuracy in predicting the relative energies is probably better than $1 \mathrm{kcal} / \mathrm{mol}$.

The specific effects due to hydrogen bond formation are much more difficult to analyze. As discussed above, errors may arise due to anharmonicities of HB vibrations. Even more complicated is the problem of choosing suitable reference structures while comparing the relative stabilities of alcohol complexes of different stoichiometry with the experimental data obtained for bulk alcohols. Related to this is another challenge for the theory, predicting the fractions of various types of alcohol complexes present in bulk alcohol solutions, in particular the contributions from different stoichiometries of the syn rotamer. It has been shown that molecular dynamics simulations can be helpful in this respect $[15,44]$. Our current efforts focus on the detailed determination of the structure of water and alcohol complexes of numerous azaaromatic compounds isolated in supersonic jets [22, 45].

An intriguing question that remains to be answered is why the formation of independent hydrogen bonds in the anti species does not lead to efficient $S_{1}$ depopulation. Such an effect could have been anticipated for chromophores which undergo a large rearrangement of electronic density upon excitation. The electronic redistribution should induce strong changes in the HB strength in $S_{1}$ with 
regard to $S_{0}$, a condition often leading to an enhancement of the internal conversion to the ground state. Actually, the fluorescence lifetimes of the anti forms, observed in alcohols, are usually longer than those of the syn rotamers, measured in acetonitrile. The longer lifetimes may be partially due to somewhat smaller radiative constants in the anti forms, an effect suggested by calculations. Anyway, no general effect of quenching by alcohol was detected for the anti forms. It seems that this phenomenon can be strongly structure-specific. For instance, shortening of the lifetime in alcohol with respect to the value obtained in polar aprotic solvents, has been observed for 7-(4'-pyridyl)indole [20], but not for its isomer, 2-(4'-pyridyl)indole [24].

\section{Acknowledgments}

The work was supported, in part, by the grants of the State Committee for Scientific Research nos. 1T09A 01830 and 1T09A 016 30. M.K. acknowledges a Ph.D. grant of the Polish Academy of Sciences and R.P.T. thanks the Robert A. Welch Foundation (E-621) and the NSF (CHE-0714751).

\section{References}

[1] N. Mataga, Y. Kaibe, M. Koizumi, Nature 175, 731 (1955).

[2] D. Rehm, A. Weller, Isr. J. Chem. 8, 259 (1970).

[3] T. Yatsuhashi, H. Inoue, J. Phys. Chem. A 101, 8166 (1997).

[4] T. Yatsuhashi, Y. Nakajima, T. Shimada, H. Inoue, J. Phys. Chem. A 102, 3018 (1998).

[5] Z. Miskolczy, L. Biczók, I. Jablonkai, Chem. Phys. Lett. 440, 92 (2007).

[6] L. Biczók, P. Valat, V. Wintgens, Phys. Chem. Chem. Phys. 1, 4759 (1999).

[7] L. Biczók, T. Bérces, H. Linschitz, J. Am. Chem. Soc. 119, 11071 (1997).

[8] L. Biczók, T. Bérces, H. Inoue, J. Phys. Chem. A 103, 3837 (1999).

[9] J. Herbich, W. Rettig, R.P. Thummel, J. Waluk, Chem. Phys. Lett. 195, 556 (1992).

[10] J. Herbich, C.Y. Hung, R.P. Thummel, J. Waluk, J. Am. Chem. Soc. 118, 3508 (1996).

[11] J. Herbich, J. Dobkowski, R.P. Thummel, V. Hegde, J. Waluk, J. Phys. Chem. A 101, 5839 (1997).

[12] J. Dobkowski, J. Herbich, V. Galievsky, R.P. Thummel, F.Y. Wu, J. Waluk, Ber. Bunsen-Ges. Phys. Chem. Chem. Phys. 102, 469 (1998).

[13] A. Kyrychenko, J. Herbich, M. Izydorzak, M. Gil, J. Dobkowski, F.Y. Wu, R.P. Thummel, J. Waluk, Isr. J. Chem. 39, 309 (1999).

[14] A. Kyrychenko, J. Herbich, M. Izydorzak, F. Wu, R.P. Thummel, J. Waluk, J. Am. Chem. Soc. 121, 11179 (1999).

[15] A. Kyrychenko, Y. Stepanenko, J. Waluk, J. Phys. Chem. A 104, 9542 (2000).

[16] D. Marks, H. Zhang, P. Borowicz, J. Waluk, M. Glasbeek, J. Phys. Chem. A 104, 7167 (2000). 
[17] J. Herbich, M. Kijak, A. Zielińska, R.P. Thummel, J. Waluk, J. Phys. Chem. A 106, 2158 (2002).

[18] J. Waluk, Acc. Chem. Res. 36, 832 (2003).

[19] M. Kijak, A. Zielińska, C. Chamchoumis, J. Herbich, R.P. Thummel, J. Waluk, Chem. Phys. Lett. 400, 279 (2004).

[20] G. Wiosna, I. Petkova, M.S. Mudadu, R.P. Thummel, J. Waluk, Chem. Phys. Lett. 400, 379 (2004).

[21] M. Kijak, E. Nosenko, A. Singh, R.P. Thummel, J. Waluk, J. Am. Chem. Soc. 129, 2738 (2007); M. Kijak, E. Nosenko, A. Singh, R.P. Thummel, B. Brutschy, J. Waluk, J. Mol. Struct. 844-845, 286 (2007).

[22] Y. Nosenko, M. Kunitski, R.P. Thummel, A. Kyrychenko, J. Herbich, J. Waluk, C. Riehn, B. Brutschy, J. Am. Chem. Soc. 128, 10000 (2006).

[23] A. Kyrychenko, J. Waluk, J. Phys. Chem. A 110, 11958 (2006).

[24] A. Kyrychenko, J. Herbich, F. Wu, R.P. Thummel, J. Waluk, J. Am. Chem. Soc. 122, 2818 (2000).

[25] I. Petkova, M.S. Mudadu, A. Singh, R.P. Thummel, I.H.M. van Stokkum, W.J. Buma, J. Waluk, J. Phys. Chem., DOI: 10.1021/jp0735841.

[26] F. Wu, J. Hardesty, R.P. Thummel, J. Org. Chem. 63, 4055 (1998).

[27] M. S. Mudadu, A. Singh, R.P. Thummel, J. Org. Chem. 71, 7611 (2006).

[28] T. Grandi, F. Sparatore, A. Sparatore, Il Farmaco 54, 479 (1999).

[29] Q.-D. Liu, M.S. Mudadu, R.P. Thummel, Y. Tao, S. Wang, Adv. Funct. Mater. 15, $143(2005)$

[30] Q. Liu, M.S. Mudadu, H. Schmider, R.P. Thummel, Y. Tao, S. Wang, Organometallics 21, 4743 (2002).

[31] M.J. Frisch, G.W. Trucks, H.B. Schlegel, G.E. Scuseria, M.A. Robb, J.R. Cheeseman, J.A. Montgomery, Jr., T. Vreven, K.N. Kudin, J.C. Burant, J.M. Millam, S.S. Iyengar, J. Tomasi, V. Barone, B. Mennucci, M. Cossi, G. Scalmani, N. Rega, G.A. Petersson, H. Nakatsuji, M. Hada, M. Ehara, K. Toyota, R. Fukuda, J. Hasegawa, M. Ishida, T. Nakajima, Y. Honda, O. Kitao, H. Nakai, M. Klene, X. Li, J.E. Knox, H.P. Hratchian, J.B. Cross, C. Adamo, J. Jaramillo, R. Gomperts, R.E. Stratmann, O. Yazyev, A.J. Austin, R. Cammi, C. Pomelli, J.W. Ochterski, P.Y. Ayala, K. Morokuma, G.A. Voth, P. Salvador, J.J. Dannenberg, V.G. Zakrzewski, S. Dapprich, A.D. Daniels, M.C. Strain, O. Farkas, D.K. Malick, A.D. Rabuck, K. Raghavachari, J.B. Foresman, J.V. Ortiz, Q. Cui, A.G. Baboul, S. Clifford, J. Cioslowski, B.B. Stefanov, G. Liu, A. Liashenko, P. Piskorz, I. Komaromi, R.L. Martin, D.J. Fox, T. Keith, M.A. Al-Laham, C.Y. Peng, A. Nanayakkara, M. Challacombe, P.M.W. Gill, B. Johnson, W. Chen, M.W. Wong, C. Gonzalez, J.A. Pople, Gaussian 03, Revision B.04., Gaussian Inc., Pittsburgh, PA, 2003.

[32] M. Wong, K. Wiberg, M. Frisch, J. Am. Chem. Soc. 114, 1465 (1992).

[33] M. Wong, K. Wiberg, M. Frisch, J. Am. Chem. Soc. 114, 523 (1992).

[34] M. Wong, K. Wiberg, M. Frisch, J. Chem. Phys. 95, 8991 (1991).

[35] M. Wong, M. Frisch, K. Wiberg, J. Am. Chem. Soc. 113, 4776 (1991).

[36] L. Onsager, J. Am. Chem. Soc. 58, 1486 (1936). 
[37] M. Cossi, V. Barone, R. Cammi, J. Tomassi, Chem. Phys. Lett. 255, 327 (1996).

[38] V. Barone, M. Cossi, J. Tomasi, J. Chem. Phys. 107, 3210 (1997).

[39] G. Almerindo, D. Tondo, J.J. Pliego, J. Phys. Chem. A 108, 166 (2004).

[40] S. Simon, M. Duran, J. Dannenberg, J. Chem. Phys. 105, 11024 (1996).

[41] S. Boys, F. Bernardi, Mol. Phys. 19, 553 (1970).

[42] E. Lim, J. Phys. Chem. 90, 6770 (1986).

[43] M. El-Sayed, J. Chem. Phys. 38, 2834 (1963).

[44] S. Mente, M. Maroncelli, J. Phys. Chem. A 102, 3860 (1998).

[45] E. Nosenko, A. Kyrychenko, R.P. Thummel, J. Waluk, B. Brutschy, J. Herbich, Phys. Chem. Chem. Phys. 9, 3276 (2007).

[46] M. Kijak, W.J. Buma, J. Waluk, in preparation. 